\title{
Antibiotic resistance in enterobacteria from pigs
}

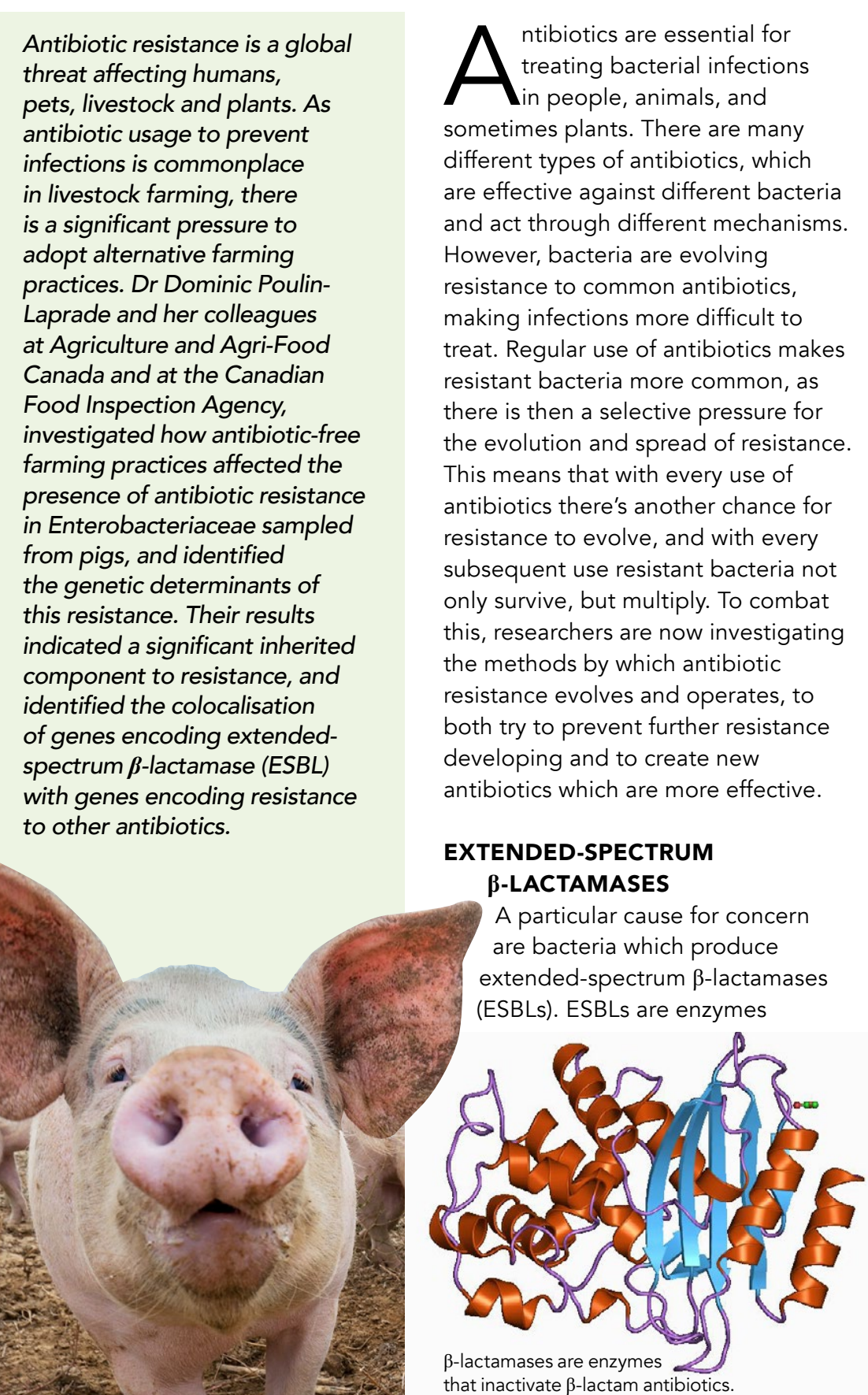

Antibiotic resistance is a glob pets, livestock and plants. antibiotic usage to prevent . is a significant pressure to practices Dr Dominic Pou identified this resistance. Their results ont the resistance, and spectrum $\boldsymbol{\beta}$-lactamase (ESBL) to other antibiotics. which confer resistance to a group of antibiotics known as $\beta$-lactams, and These are two lin and cephalosporins antibiotics used to trenta wide variety of infections, and third-generation cephalosporins (3GC) such as cefotaxime (CTX) are broad-spectrum antibiotics considered very important in medicine. ESBL-producing bacteria are therefore considered a serious health threat.

\section{ANTIBIOTICS IN LIVESTOCK} Antibiotics are used in vast quantities in livestock farming to prevent infections and ensure that potentially harmful bacteria are not passed from animals to people through meat or manure used on crops. However, this extensive antibiotic use is thought to escalate the development of antibiotic resistant bacteria. Organic farming treatment of sick animals (as opposed to conventional farms using antibiotic prophylaxis), and are increasing in popularity as a result of increasing awareness of antibiotic resistance. Dr Dominic Poulin-Laprade and her colleagues at Agriculture and AgriFood Canada and at the Canadian

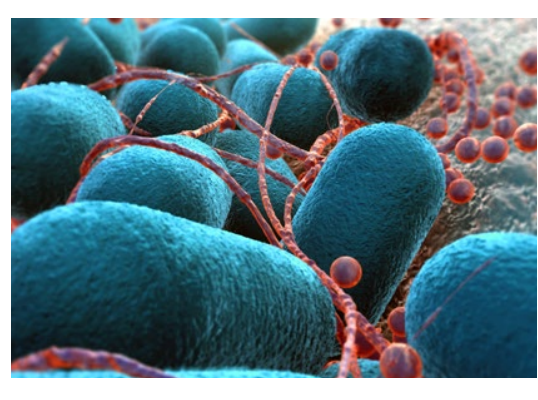

Bacteria evolve resistance to common an
making infections more difficult to treat.
Food Inspection Agency, investigated whether rearing pigs without the the quantities of antibitic resistant Enterobacteriaceae (gram-negative bacteria including $E$ coll found in the porcine intestinal microbiota.

\section{ANTIBIOTIC-FREE PIG FARMING} Pigs are one of the three largest live
groups in Canada (with cattle and groups in Canada (with cattle and bacteria which canown to carry resistant through tainted meat and manure. To investigate the effect of swine farming methods on the prevalence of antibiotic resistance, Dr Poulin-Laprade and colleagues took samples from three different antibiotic-free farms and one conventional (CV) livestock farm. The first of the antibiolicfiee farm types (AFI) certified humane, and the third (AF3) certified AGRO-COM. AF1, AF2 and AF3 met the requirements of the 2 and $A F 3$ Food Inspection Agency allowing the display of the antibiotic free claim on their marketed products.

\section{ANTIBIOTIC RESISTANCE IN PIGS} antibiotic resistance was less common in antibiotic-free farming practices, supporting the theory that continual antibiotic use results in the spread of antibiotic resistance. It is therefore surprising that Dr Poulin-Laprade and colleagues antibiotic susceptibility testing rest ts found hact CTX resistance was more commonly found in samples Additionally, when the resistance profile of the CTX-resistant and CTX-susceptible bacteria was investigated by testing with 24 other antibiotics, ESBL-production was more common in the CTX-resistant samples from the antibiotic-free farms than from the $\mathrm{CV}$ sites. However, CTXresistant bacteria from the $\mathrm{CV}$ farms were more likely to also be resistant to other antibiotics. Importantly, they also found that across all sites the abundance of enterobacteria declined as the pigs aged, therefore lowering the antibiotic

\section{INHERITED RESISTANCE}

These results suggest that antibiotic use does increase antibiotic resistance
and creates an environment favouring
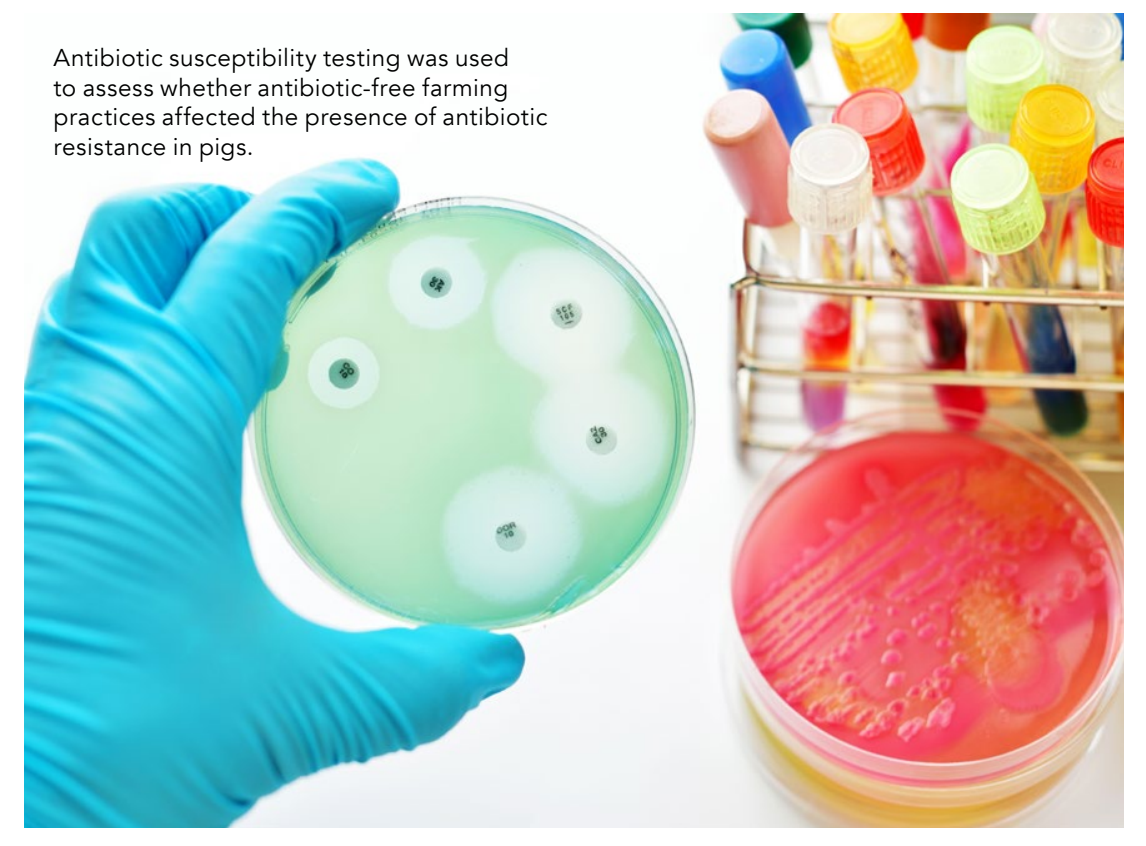

a diverse resistance profile, but that other factors such as inheritance and environment also play a role. Tetracycline resistance was less common in the antibiotic-free farms, all sites. This agrees with findings of other studies, and likely results from the 70 years of tetracycline use in people and livestock. Thus tetracycline resistant bacteria were inherited from the mother in antibiotic-free pigs, and were found to be carried by plasmids lso containing genes conferring three were associated with a genetic sequence known as ISEcp1 which is involved in the movement of antibiotic resistance genes from chromosomes to plasmids and between plasmids. The movement of genes across bacterial chromosomes and plasmids creates a continuous genetic diversity that contributes to the success of antibiotic . resistance in Enterobacteriaceae, but

The team identified three genes involved in ESBL production, the most frequent of which was only found in samples from the antibiotic-free farms.

at the antibiotic-free farms could be explained by microbiota inheritance from the sow.

\section{GENETIC DETERMINANTS} Whole genome sequencing can possessed by sampled bacteria. Plasmids are circular DNA molecules separate from the bacterial chromosome, which often carry genes involved in antibiotic resistance. The research team identified three genes involved in ESBL production, the most requent of which was only free farms. Two of these three genes colocalisation is also prevalent and contributes to multidrug resistance. It is also important to note that there are diversity of mechanisms through which bacteria manifest antibiotic resistance.

CO-SELECTION

This colocalisation of genes for with the firdistance is in agreement resistance to several antibiotics when the prevalence of the gend explain many factors which work together to

\section{COLOCALISATION AND} with the findings from other studies, and will lead to co-selection for 


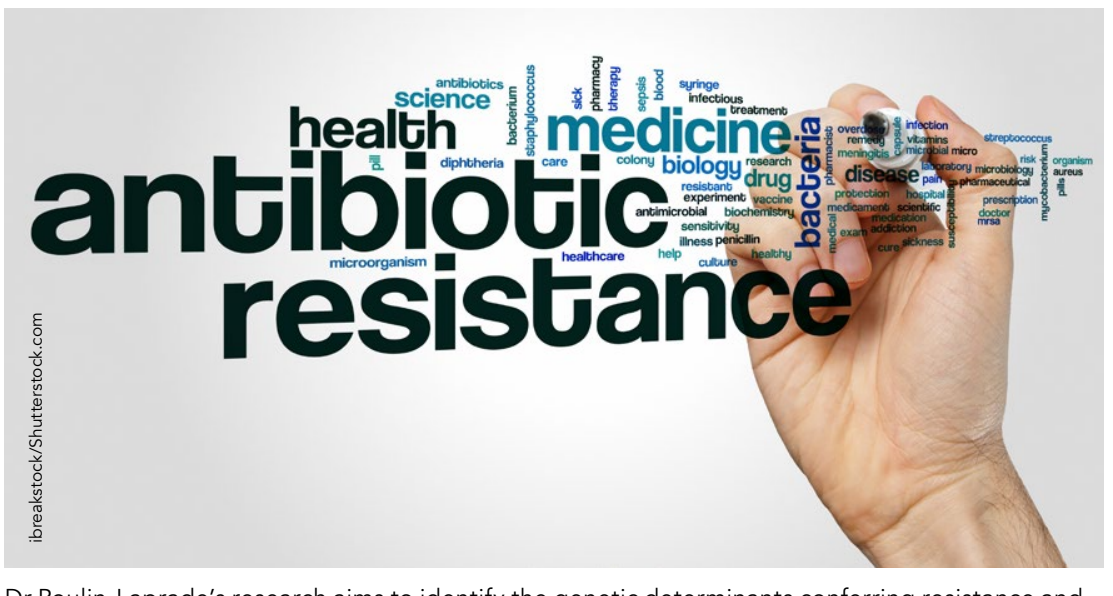

Dr Poulin-Laprade's research aims to identify the genetic determinants conferring resistance an
develop mitigating strategies to reduce antimicrobial resistance in pig production.

Dr Poullin-Laprade suggests that intervention at the level of breeding farms could be complementary to antibiotic stewardship and more effective in reducing antibiotic resistance determinants that are passed down by the sow.

production in the antibiotic-free conditions, as the most common of those genes is colocalised and coselected with genes for resistance to antibiotics commonly used as treatment in diseased pigs in Canad. Another such finding from this study was that a gene for a penicillinase (an enzyme that acts against penicillin) was colocalised with genes conferring resistance to heavy metals such as copper, and the in the pig feed copp contribute to the maintenance of the cenicillinase genes.

\section{CONJUGATION} plasmids through a process known

and colleagues found a plethora of conjugative plasmids harbouring resistance genes, and testing showe that transference of CTX resistance and ESBL-encoding genes was possible in their sampled bacteria and unaffected by whether or not antibiotics were used in the farming process. They therefore hypothesis that the high levels of CTX resistance in the antibiotic-free sites could be due to the gene being present through conjugation. Additionally, the absence of the most common ESBL-encoding gene in the CV sites could be due to these farms being independent - if no animals are transferred then the enterobacteria present may never have encountered mobilising plasmid.

ACTION AGAINST ANTIBIOTIC RESISTANCE Overall, Dr Poulin-Laprade and colleagues showed that the genetic component of antibiotic resistance is a major factor that is not easily combatted, as evidenced by the presence of antibiotic resistance genes in bacteria sampled from pig farms where antibiotics aren't used. However, antibiotic susceptibility testing found that the bacteria from the antibiotic-free sites were on average resistant to fewer antibiotics, due to a lower selective pressure. This could suggest that Prganic farming may help reduce the many years of widespread antibiotic prophylaxis in livestock. On the

other hand, Dr Poulin-Laprade and

colleagues suggest that intervention at the level of breeding farms could be complementary to antibiotic stewardship and more effective in reducing antibiotic resistance determinants that are passed down by the sow.

Additionally, Dr Poulin-Laprade and colleagues emphasise that there is a serious environmental risk from

antibiotic resistance. Often, antibiotics can pass through animals and still be This not gainst bacteria in the soil. This not only impacts us when tainted manure is used on crops which we eat, the soil, resulting in poor plant growt and resilience. With climate change an ever-present threat, a balance needs to be found for protecting food supply in terms of large, healthy crop yields, and in terms of ensuring the health of livestock.
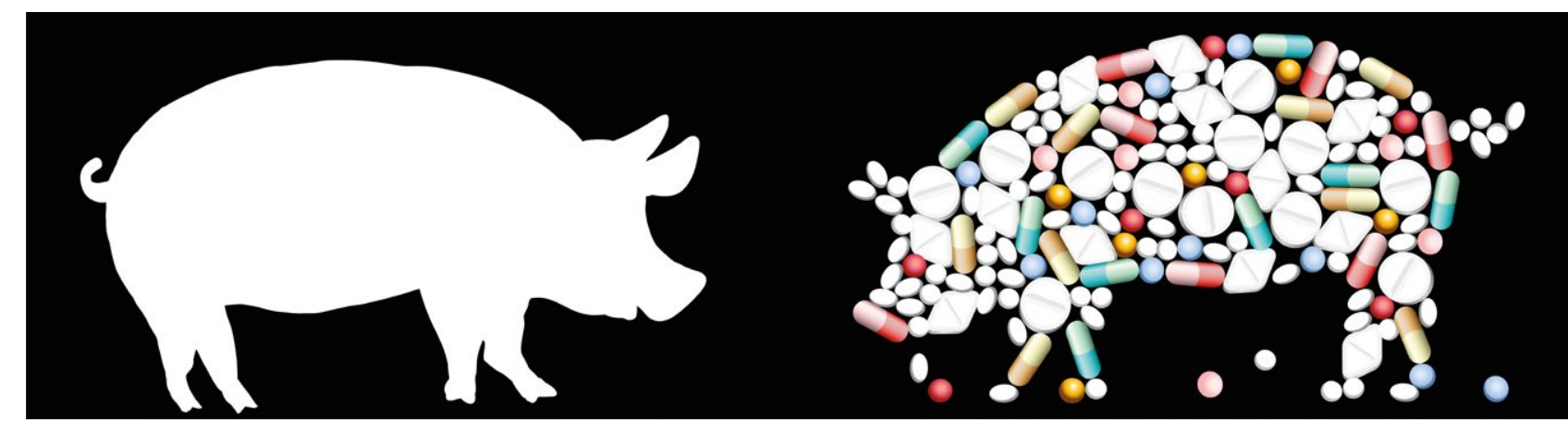

E: dominic.poulin-laprade@agr.gc.ca $\quad$ T: +1 819-571-0864

W: https://profils-profiles.science.gc.ca/en/profile/dominic-poulin-laprade-phd

\section{Research Objectives}

Dr Poulin-Laprade's research aims to identify the genetic determinants conferring resistance and develop mitigating strategies to reduce antimicrobial resistance in pig production.

\section{Detail}

Sherbrooke Research and Development Centre Agriculture and Agri-Food Canada 2000 College Street

Sherbrooke, Quebec J1M OC8

Bio

Dr Poulin-Laprade is a researcher at AAFC and an adjunct professor at the University of Sherbrooke and at the Laval University. Her primary expertise lies in bacterial genetics, horizontal gene transfer, and antimicrobial resistance. In recent years she has acquired additional expertise in pig production, pig gut microbiome and immune system.

Funding

mics Research \& Development Initiative on Antimicrobial Resistance (GRDI-AMR)

\section{Collaborators}

From Agriculture and Agri-Food Canada: - Guylaine Talbot - Microbial Ecology

- Edward Topp - Environmental microbiology

and chemistry

- Tim A. McAllister - Ruminant Nutrition \& Microbiology - J. Jacques Matte - Swine nutritio

From the Canadian Food Inspection Agency:

Catherine D. Carrillo - Method development for food microbiology laboratories

\section{Reference}

Poulin-Laprade, D Brouard, JS Gagnon, N Turcotte, A Langlois, A Matte, JJ Carrillo, CD Zaheer, R McAllister, TA Topp, E and Talbot, G (2021). Resistance Determinants a Longitudinal Study of Pigs Reared under Various Husbandry Conditions. Applied and Environmental AEM.02612-20

\section{Personal Response}

In your opinion, how effective are antibiotic-free farming practices at red
the microbiota of pigs?

II Antibiotic-free practices do lower the frequency of some antibiotic resistance genes in the microbiota those that are relevant sufficient to eliminate all as observed with the persistence of bla $a_{\text {CT-M-M-l }}$ in the antibiotic-free herds we studied. Resistant bacteria
can have an increased fitness and thrive due to factors applied by antibiotics. For example, they can be ane to bacteriophages that infect and control microorganisms of the microbiota and be tolerated by the pig immune system. Reducing the use of resistance is a complex issue that needs to be fought with a diverse arsenal on numerous fronts along the
One Health continuum.

\section{Canadà} other than their resistance to the selective pressure antibiotics is part of the solution, but antimicrobial 